\title{
LA TOMA DE DECISIONES DE DIRECTIVOS LATINOS
}

Michel Fiol

Profesor de Management y Control de Gestión de la HEC School of Management.

E-mail: fiol@hec.fr

\section{RESUMEN}

Dos dificultades ligadas a la presión del tiempo pertuban la toma de decisiones de un directivo en una empresa. Los datos y los conocimientos que el management "moderno" pone a su alcance, a su disposición, son cada vez más numerosos y le desbordan; por outro lado, su necessidad de decidir rápidamente, dada su obligación de dar resultados a muy corto plazo, le deja muy poco tiempo para pensar. El objectivo de este artículo es verificar la toma de decisiones de directivos latinos y compararla a la de los directivos norteamericanos. Por esta razón, hemos decidido recurrir a una situación compleja en la que hay que tomar una decisión. Un caso fue presentado a directivos de Europa del Sur, de América Latina y, posteriormente, a directivos latinos y norteamericanos de empresas multinacionais. Hemos detectado que, a diferencia de los latinos, los norteamericanos toman su decisión en muy poco tiempo, aplicando, sin vacilar y de forma estricta, reglas éticas compartidas.

\section{ABSTRACT}

Time pressure imposes two major dilemmas on managers' decision-making processes. On the one hand, "modern" management translates into increasing knowledge and amount of data available; on the other hand, the fast pace required for achieving short-term results leaves managers very little time to think. The aim

of this article is to analyze the decision-making process of Latin managers and to compare it with their American counterparts. Therefore, we have decided to create a complex situation in which a decision has to be made. A case was presented to managers from South Europe and Latin America and, later on, to Latin and American managers of multinational companies. The results showed that, unlike the Latin colleagues, the Americans decide quickly and apply, in a very strict manner and without hesitation, the shared ethical rules.

\section{PALABRAS CLAVE}

Toma de decisiones, directivos latinos, directivos norteamericanos, situaciones complejas, mentalidad-solución.

KEY WORDS

Decision-making process, Latin managers, American managers, complex situations, "solution" approach. 


\section{INTRODUCCIÓN}

Dos dificultades ligadas a la presión del tiempo perturban la toma de decisiones de un directivo en una empresa. Los datos y los conocimientos que el management "moderno" pone a su alcance, a su disposición, son cada vez más numerosos y le desbordan; por otro lado, su necesidad de decidir rápidamente, dada su obligación de dar resultados a muy corto plazo, le deja muy poco tiempo para pensar.

Por una parte, los nuevos sistemas de información, que proponen medir todo dentro de la empresa, satisfacen teóricamente el sueño de todo decisor racional. ¿Quién no aspira a tener a su alcance todos los datos necesarios, poder explorarlos y estudiarlos exhaustivamente antes de decidir, con el fin de tomar las decisiones "óptimas"? Sin embargo, la mente humana tiene una capacidad limitada para estudiar y combinar un gran número de datos ${ }^{1}$. La observación de situaciones reales en las que un directivo debe decidir individualmente o en grupo cuando dispone de mucha información muestra que, contrariamente a lo que se espera, el directivo no toma una decisión siguiendo un proceso racional (lo que no significa que adopte un método irracional); además, desestabilizado por el exceso de información, opta a menudo por "no decidir". En ninguno de los dos casos, el decisor no consigue procesar la gran cantidad de información de que dispone.

Por otro lado, bajo la presión de los acontecimientos y ante las exigencias de la urgencia, el directivo no se toma el tiempo necesario para desarrollar métodos reflexivos, comprender las situaciones complejas a las que se enfrenta, dándose así la posibilidad de detectar conexiones no aparentes entre sus diferentes aspectos. En el mejor de los casos, busca identificar relaciones de causa-efecto entre dos factores para explicar estas situaciones y decide tratar la variable independiente para influir sobre la dependiente. Generalmente, opta por una solución entre dos o tres de las posibles y tiende a justificar su decisión a posteriori. La experiencia, el contacto permanente con situaciones concretas, los fracasos y los éxitos, la repetición de las situaciones conflictivas o contradictorias son factores que influyen en la selección de la decisión tomada.

Sin embargo, la observación de las consecuencias que tuvieron decisiones reales no permite establecer comparaciones en el tiempo y en el espacio, ya que nunca se presentan de la misma forma. Por esta razón, hemos decidido recurrir a una situación experimental: hemos elaborado un caso describiendo una situación compleja en la que hay que tomar una decisión (incluyendo la posibilidad de no decidir). En una primera etapa, hemos sometido este caso al análisis de numerosos directivos de empresas en Europa del Sur y en América Latina. Hemos observado que estos directivos aprovechan todo el tiempo del que dispongan para decidir y que manifiestan siempre los mismos reflejos cognoscitivos o mentales. En una segunda etapa, hemos aplicado el mismo caso en dos grandes empresas multinacionales contando con grupos separados de directivos latinos y norteamericanos. Hemos detectado que, a diferencia de los latinos, los norteamericanos toman su decisión en muy poco tiempo, aplicando, sin vacilar y de forma estricta, reglas éticas compartidas.

\section{EL PRIMER EXPERIMENTO}

El dispositivo experimental construido para comprender como toman mentalmente decisiones los directivos utiliza un caso, inspirado en una situación real de empresa.

Este caso $^{2}$ presenta la problemática compleja a la que se enfrenta un jefe de división en una empresa que cuenta con cuatro divisiones. A petición del director general, se deberá alcanzar un objetivo de rentabilidad que el jefe de división considera inalcanzable. Ante la obstinación del director general, y después de haber resistido un poco, acepta el reto y lo impone a sus colaboradores inmediatos.

\section{LOS NUEVOS SISTEMAS DE}

\section{INFORMACIÓN, QUE PROPONEN MEDIR TODO DENTRO DE \\ LA EMPRESA, SATISFACEN TEÓRICAMENTE EL SUEÑO DE TODO DECISOR RACIONAL.}

Éstos, a su vez, manifiestan en vano su inconformidad con la meta impuesta y terminan por aceptar elaborar un plan y un presupuesto para alcanzar el objetivo deseado. Como no logran "por las buenas" tener los resultados esperados, los colaboradores deciden, a espaldas de su superior jerárquico, recurrir a métodos menos ortodoxos: el responsable de ventas impone, de diferentes maneras, ventas forzadas a sus clientes y el responsable de producción manipula las facturas, mediante previo acuerdo con sus proveedores, para transformar gastos en activos amortizables. El contralor de la división no se percata de estas indelicadezas y malversaciones. Después de luchar durante varios meses con sus métodos deshonestos para alcanzar los resultados y empezando a temer los posibles efectos desastrosos de sus iniciativas, los dos responsables de ventas y de producción se reúnen con el jefe de la división y le cuentan todo. El jefe de división se pregunta qué hacer, sabiendo que el director general piensa que él no tiene suficiente capacidad para 
asumir su cargo.

Como podemos observar, la situación presentada es muy compleja ya que mezcla aspectos estratégicos, organizacionales, económicos, éticos, sociológicos y psicológicos, etc. El caso fue sometido en una primera etapa $^{3}$ a más de 700 directivos de Europa del Sur y de América Latina, esencialmente argentinos, brasileños, franceses y mexicanos, tres cuartas partes de los cuales trabajaban en grandes empresas y el cuarto restante lo hacían en pequeñas y medianas empresas. Todos ellos, con perfiles educacionales y profesionales diferentes, seguían programas de educación permanente.

En un primer tiempo, se pedía a los diferentes grupos responder a la pregunta siguiente: "Pónganse en el papel del director de división. Acaban de recibir las confesiones de los responsables de ventas y de producción. ¿Qué harían frente a esta situación compleja?" Se les daba un tiempo limitado para contestar a la pregunta, variando el tiempo de reflexión de una a cuatro horas. Se observaba el trabajo de grupo y se estudiaba el método que seguían para llegar a la decisión final. Una vez tomada la decisión se pedía a los directivos, en un segundo tiempo, que analizaran el proceso cognoscitivo individual y colectivo que habían seguido para llegar a dicha decisión. Se pedía al final a cada grupo que presentara a sus homólogos el resultado de su discusión y el método mental utilizado.

Los principales resultados obtenidos son los siguientes:

- Cuando trabajan en grupo, utilizan todo el tiempo disponible para discutir el caso (de una a tres horas).

- Las respuestas aportadas no dependen del tiempo de discusión que se les haya dado.

- Los directivos latinos, cualquiera que sea su país de origen, adoptan siempre esquemas mentales similares. Cuatro de ellos son particularmente frecuentes: el desconcierto metodológico, la mentalidad-solución, la lógica del conflicto de opiniones y la parálisis de decisión.

- A excepción de la lógica del conflicto de opiniones, los otros tres reflejos mentales se manifiestan cualquiera que sea el número de directivos implicados en el proceso de decisión: de una a cuatro personas.

- Cuando los directivos toman su decisión en grupo, opera la lógica del conflicto de opiniones; pero, al final de la discusión, los grupos casi siempre llegan a un consenso y manifiestan una cierta satisfacción de haberlo logrado.

- Los comportamientos generados por el estudio del caso se pueden clasificar en dos grandes categorías: Alrededor de $80 \%$ de los grupos aportan soluciones, muy diferentes entre si. El 20\% restante sugiere crear un comité para analizar la situación, ya que ellos se sienten incapaces de hacerlo, debido al tiempo limitado y a considerar que disponen de insuficiente información.
- En todos los casos, se considera que el objetivo fijado por la dirección general es inalcanzable.

- Los dilemas más comunes son: a) reunirse inmediatamente con el director general y contarle lo que pasa, o bien darse el tiempo de enderezar la situación antes de informarle; b) manifestar una buena capacidad administrativa, revisando a la baja los objetivos y elaborando un plan realista, o bien renunciar; c) renunciar definitivamente, o proponer su renuncia con la soga al cuello esperando que el director general no se atreverá a apretarla; d) denunciar a sus colaboradores o encubrirles; e) designar un chivo expiatorio y despedirle para dar el ejemplo, o solamente amonestarles; f) despedir a alguien, pero ¿a quién? ¿Al responsable de ventas, al responsable de producción o al contralor de gestión?

- En las discusiones y presentaciones de los trabajos, se utiliza muy a menudo el término "problema". Cuando se quiere hablar de un malestar, una falta de ética, una dificultad, una diferencia entre lo real y lo previsto, una disfunción, un error, un resultado insuficiente, una situación delicada, etc., se dice indistintamente que "hay un problema". Pero lo paradójico es que, cuando se aportan soluciones, éstas no corresponden a ningún problema identificado formalmente.

- Las soluciones concretas más comúnmente propuestas son las siguientes: hacer una auditoría de la situación, proceder a una restucturación de los procesos de compra, mejorar el sistema de control de gestión, crear un departamento de auditoría interna, elaborar una carta de principios éticos para la compañía, renunciar, despedir a uno de los colaboradores o exigir del director general que revise a la baja sus objetivos.

- Se observa que estas soluciones son muy diferentes y que tienden a responder a problemas diferentes. Cuando se pregunta a cada grupo cuál es el problema al que responde la solución propuesta, uno descubre que el grupo induce el problema a partir de la solución que aporta. Los que han propuesto elaborar una carta de principios éticos afirman que el problema principal de la compañía es la falta de ética; los que recomiendan mejorar el sistema de control de gestión declaran que existe un problema de control de gestión; los que sugieren renunciar consideran que tienen un "verdadero" problema de liderazgo, etc.

- Para justificar los despidos de los colaboradores, los directivos no aplican principios éticos definidos $a$ priori, sino aquéllos que aparecen en el transcurso de las discusiones. Los directivos recurren a dichos principios para defender su posición o para destruir la de otro miembro de su grupo. Así, para despedir al responsable de ventas, al responsable de producción y al contralor de gestión se proponen respectivamente los postulados siguientes: debe renunciar toda persona que deteriora la imagen de la empresa ante los clien- 
tes; debe ser castigado todo colaborador que comete un delito; debe ser despedido todo individuo que no cumple con sus obligaciones de trabajo y, por lo tanto, no es útil (se considera entonces que los responsables de ventas y de producción por lo menos han intentado actuaren favor de la empresa).

Se presentan a continuación los cuatro reflejos mentales más comunes cuando directivos latinos, confrontados con una situación compleja, deben tomar una decisión.

\section{LOS CUATRO REFLEJOS}

\section{COGNOSCITIVOS MÁS COMUNES ${ }^{4}$}

El "desconcierto metodológico" es el reflejo mental más común entre los directivos latinos. Frente a una situación de difícil decisión, éstos sienten una gran dificultad para desarrollar un modo elemental de razonamiento "estructurante". La necesidad de poner orden en su mente cuando se enfrentan a una dificultad imprevista, no habitual o extraña, incita a los directivos a recurrir a un mínimo de método formal racional. Se observa frecuentemente la voluntad de utilizar uno de los dos procesos de razonamiento siguientes: una relación lineal sencilla del tipo "diagnóstico de la situación $\rightarrow$ identificación del problema $\rightarrow$ censo y análisis de diferentes soluciones $\rightarrow$ selección de una solución", o bien un árbol de decisiones.

El método preferido por los directivos consiste en adoptar un proceso de decisión en cuatro fases: a) hacer un diagnóstico de la situación, es decir describirla lo más ampliamente posible (¿de qué se trata? ¿qué pasa "realmente"?) e identificar o, por lo menos, circunscribir lo que no funciona correctamente (¿qué anda mal y por qué?), con la intención de que el análisis sea lo más objetivo posible; b) definir, en consecuencia con el resultado de la primera fase, el problema (¿cuál es el problema?); c) aportar dos o tres soluciones al problema y definir un criterio para seleccionar la mejor solución; d) escoger la solución que se juzgue más pertinente. Este proceso funciona como una sucesión de relaciones de tipo causaefecto. En la práctica se observa que se omite la segunda fase; los directivos pasan un cierto tiempo trabajando sobre el diagnóstico y llegan directamente a la selección de algunas posibles soluciones, saltándose la fase de identificación del problema. A veces, se eliminan las dos etapas intermedias, pasando directamente del diagnóstico a una solución.

Un segundo método consiste en construir un árbol de decisiones. Pero este método de análisis es poco utilizado porque, cuando empieza, no se termina nunca; hay un momento, cuando tienden a multiplicarse demasiado las ramas del árbol o cuando algunas de las ramas paralelas no son independientes unas de otras y se entrelazan, que este método resulta poco práctico para los directivos.

Un tercer camino mental de razonamiento podría consistir en utilizar uno de los métodos de resolución de problemas que existen en la literatura o que se dan a veces en cursos de formación continua sobre el tema. Estos métodos son muy estructurados, secuenciales; además, se proponen como sistémicos a la vez que analíticos, exhaustivos en cuanto a los factores tomados en cuenta y universales. Pero los directivos no evocan nunca estos métodos. Se debe seguramente a que: a) es imposible aplicarlos seriamente en la práctica, ya que son demasiado complicados para ser implementados fielmente; b) son esencialmente teóricos pero, al mismo tiempo, simplistas en sus fundamentos conceptuales; c) exigen pasar por un procedimiento lineal único, aplicable a todo tipo de problema o de situación; d) son demasiado ambiciosos y poco realistas, ya que excluyen a los diferentes actores involucrados en la situación.

\section{LA MENTE HUMANA TIENE}

\section{UNA CAPACIDAD LIMITADA}

\section{PARA ESTUDIAR Y COMBINAR}

\section{UN GRAN NÚMERO DE DATOS.}

El primer proceso es demasiado lineal y poco fiable, el segundo, ilusorio y el tercero, demasiado secuencial y complicado; los tres carecen de fineza y de sensibilidad. $\mathrm{Al}$ tratar de explicar la situación, sugiriendo relaciones de causa-efecto siempre simplificadoras, estos métodos se muestran insuficientes para que los directivos latinos consigan comprender la sutileza y la complejidad de las situaciones estudiadas.

El más preocupante de los reflejos mentales comunes es seguramente la "mentalidad-solución". Los directivos se proveen, con el tiempo y la experiencia, de un abanico de soluciones y aplican una de ellas en función de la representación mental que se hacen de la situación confrontada. Lo cual significa que no adaptan una solución al contexto, sino que se construyen el contexto para que sea compatible con la solución seleccionada. En otros términos, en lugar de identificar un problema y de aportar consecuentemente una solución apropiada, inducen el problema a partir de una de las soluciones que saben implementar. Frente a una situación, reaccionan con sus propios esquemas mentales, con su visión del mundo; no están influenciados por la situación externa a la que están confrontados, sino que la construyen en su mente para justificar la solución que desean aportar.

Son varios los motivos que conducen al reflejo mental de mentalidad-solución. Existe, en primer término, la 
confusión semántica alrededor del concepto de problema. Se confunden los términos de problema, situación, causa, síntoma, señal, solución, disfunción, etc. Es usual oír que hay un problema de costos cuando éstos aumentan (pero el hecho de que aumenten es más bien una señal o un síntoma); se dice que el problema es complejo (cuando es la situación que lo es) o que se trata de un problema de restructuración (se confunde entonces un posible modo de resolución con el problema). Cuando algo no funciona como debería funcionar en una situación, se dice que existe un problema; si hay un problema, se debe aportar una solución para que él desaparezca. Otro motivo es la experiencia, que actúa tanto como un factor que facilita

\section{BAJO LA PRESIÓN DE LOS}

\section{ACONTECIMIENTOS Y ANTE LAS}

\section{EXIGENCIAS DE LA URGENCIA, EL}

\section{DIRECTIVO NO SE TOMA EL TIEMPO}

NECESARIO PARA DESARROLLAR

\section{MÉTODOS REFLEXIVOS.}

la toma de decisiones, como siendo el detonador de malas decisiones. Se observa así que los directivos suelen aplicar nuevamente una solución que funcionó con éxito en otras situaciones y evitan utilizar otra que condujo anteriormente a un fracaso. La influencia de las modas gerenciales sobre el modo de pensar de los directivos es otra justificación de la mentalidad-solución. En efecto, cuando el conjunto de soluciones clásicas no sirve para hacer frente a un "problema", aparecen nuevas soluciones "milagrosas o salvadoras", cuya aplicación permite ganar tiempo, aún si no alivia satisfactoriamente la situación. Sin embargo, en estos últimos tiempos, los directivos empiezan poco a poco a darse cuenta que "demasiadas modas consecutivas matan a las modas". Un último motivo de la mentalidad-solución reside en la aplicación misma de dos famosos principios de management: a) los directivos no deben consagrar demasiado tiempo a la identificación de problemas, se les paga para aportar soluciones: b) lo que espera un directivo de sus colaboradores no es que le traigan problemas, sino soluciones.

Es probable que muchas de las soluciones propuestas no mejoren en nada las situaciones y que, eventualmente, las empeoren. Sin embargo, hay que recordar que, si bien equivocarse de solución es grave, equivocarse de problema es mucho más grave.

Y cuando se pregunta a cada grupo por qué le parece que su solución es la buena, generalmente su respuesta refleja la influencia de la experiencia ("ya hemos vivido dos veces un problema similar en mi empresa, lo resolvimos de esta forma y funcionó"), del mimetismo ("implementaron esta solución en otra división de mi compañía o en otra empresa y les resultó benéfico"), de la moda gerencial del momento ("en realidad, es un problema de restructuración o de benchmarking"), de convicciones personales ("estamos íntimamente convencidos de que el problema es éste") o de certidumbres ("el problema es evidente"), etc.

El tercer reflejo mental propuesto aquí es la lógica del "conflicto de opiniones" (y de juicios de valor). Se manifiesta de tres formas diferentes. Cada directivo se identifica con su rol, impuesto por el caso o vivido por él en su vida real de trabajo, y construye la situación a través de éste. Además, justifica a posteriori su decisión apoyándose en principios que son en realidad postulados, hipótesis y supuestos implícitos y generalmente no conscientes. Por último, se enfrentan a los que no están de acuerdo con su opinión, tratando de defenderla sin escuchar, o escuchando muy selectivamente los argumentos de sus contrincantes.

Cada directivo involucrado en el análisis de una situación tiene tendencia a identificarla con su rol dentro de la empresa (Williamson, 1970). El responsable de la mercadotecnia ve la situación a través del prisma de su disciplina, el director financiero la codifica con sus lentes de finanzas, el jefe de una división la estudia con una visión "divisional". Este reflejo es normal para cada actor dentro de una organización ya que se encuentra legitimado por la defensa de su propia responsabilidad, pero presenta el peligro de conducirle a refugiarse en una visión parcial - y, por lo tanto, estrecha - de la situación. Los directivos tienden entonces a colocarse en una lógica de conflicto de opiniones: cada uno de ellos defiende sus opiniones en contra de las de los demás y realiza juicios de valor negativos sobre las que se oponen a las suyas. Cuanto más fuertes son las convicciones de los directivos, más se amplía este fenómeno. En otras palabras, cuanto más un directivo tiende a defender sus propias ideas, más se ciega a las que difieren de las suyas.

La metáfora de los lentes (Fiol et al., 1998) es útil para ilustrar este reflejo mental (ver Figura 1). Como no hay un solo problema, sino varios mezclados e interrelacionados, no se parte del concepto de problema sino del de situación (menos ambiguo y más amplio) a la que se enfrentan diferentes personas involucradas. Cada persona se construye la situación en función de un cierto esquema mental forjado a lo largo de los años a través de la influencia familiar, la educación, la cultura, la experiencia, etc. Cada esquema mental funciona como un filtro, como unos lentes que, consciente o inconscientemente, seleccionan solamente una parte de la situación y dejan la ilusión que está siendo abarcada en su totalidad.

Cuando, con sus lentes, un directivo considera que su 
análisis de la situación es impreciso, su reflejo normal es pensar que se debe a una insuficiencia de capacidad óptica de sus lentes y que la única posibilidad que tiene de ver mejor la situación es reforzar esta capacidad. Pero al concentrar más su mirada sobre la parte de la situación que ha seleccionado naturalmente, la persona se vuelve más ciega a las demás partes que no capta (ver Figura 2). Se produce entonces una focalización excesiva hacia lo visto y una exclusión peligrosa de lo no visto. En otras palabras, se tiene la ilusión de que se está analizando mejor una situación, cuando en realidad lo que sucede es justo lo contrario.

La metáfora de los lentes ayuda a buscar una alternativa a la mentalidad-solución:

a) Tomar consciencia de sus propios lentes (o sea, de su propio esquema) y de la estrechez de su campo de visión; es decir, darse cuenta de que cada uno construye su propia realidad, y de cómo lo hace.

b) Considerar y aceptar que hay otros lentes que analizan a su manera la situación, cada uno con su ángulo de entrada, teniendo todos ellos visiones sesgadas pero complementarias entre sí.

c) Aceptar que una mejor comprensión de la situación pasa, no por la mejora de la capacidad óptica de sus propios lentes (lo que tiene el peligro de conducir a "más y mejor de lo mismo"), sino por una confrontación de los diferentes puntos de vista.

d) Crear las condiciones para que esta confrontación de puntos de vista sea efectiva y fructífera.

En otros términos, la metáfora de los lentes implica tres cambios de actitud. Se debe pasar:

- de un conflicto de opiniones (en donde se trata - en un juego algunas veces duro, pero generalmente sutil -, de defender su opinión, rebatir la de los demás y convencerles de que la propia opinión es la mejor) a

\section{Figura 1 - Metáfora de los lentes}

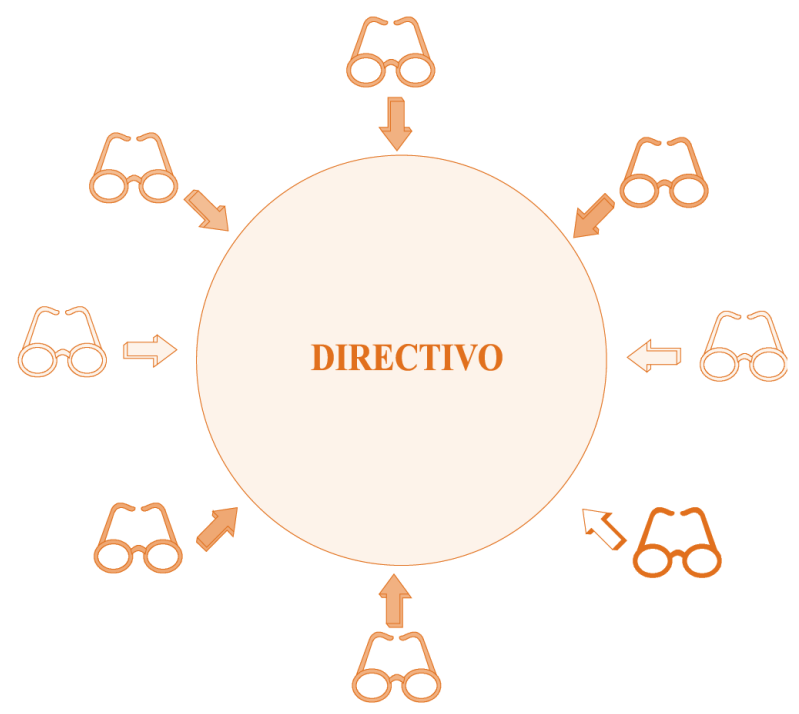

una confrontación de puntos de vista (en la que cuanto más diferentes sean los puntos de vista de los demás con respecto al suyo, más interesante será, ya que aportarán otras miradas que pueden enriquecer la comprensión de la situación);

- de la utilización de capacidades personales (un estudio individual, parcial, partidario, de las situaciones) al desarrollo progresivo de competencias colectivas (un aprendizaje de la detección y del reconocimiento de los actores afectados por la situación, de la aceptación y del respeto de puntos de vista diferentes y del enriquecimiento colectivo por la confrontación de éstos);

- de la perspectiva del "o" (toda negociación entre dos partes se hace sobre la base de una transacción: lo que gana una parte lo pierde la otra y viceversa) a la del "y" (las dos partes buscan de forma creativa y colectiva una manera original de hacer frente a la situación en la que se integran los puntos de vista, respetándose y complementándose mutuamente).

Es interesante observar que los directivos tienen una visión muy estrecha de la situación estudiada y, en consecuencia, un reconocimiento cerrado de los actores involucrados en ella; tienden a tomar en consideración únicamente a los actores situados en su entorno inmediato, o sea, muy cercanos a ellos, directamente implicados en la situación e internos a su empresa. Raras veces se preocupan por actores externos a su empresa, como son los clientes, los proveedores, los competidores, los auditores externos, etc.

La identificación con su rol no es la única manifestación de la lógica de los conflictos de opiniones. Otra consiste en apoyarse inconscientemente en imperativos, postulados, hipótesis o supuestos implícitos para justificar una decisión. Por ejemplo, en el caso estudiado, uno de los personajes considera que le va a ser imposible alcanzar el resultado que le exige su superior jerárquico; los directivos parten inconscientemente del principio de que el resultado es inalcanzable y lo adoptan como un postulado sin discutirlo, sin aún evocarlo. Otro ejemplo es el de la búsqueda, por parte de los directivos, de un personaje del caso que sirva de chivo expiatorio, acusándole de la entera responsabilidad de los malos manejos ocurridos en la empresa. Tres personajes pueden ser despedidos para "dar ejemplo"; el gerente de ventas, el de producción y el contralor de gestión. Para eso, el primero es acusado, por los directivos que desean despedirle, de dañar la imagen de la empresa frente a sus clientes (es imperativo para ellos que la empresa cuide a toda costa la imagen que da a sus clientes); al segundo se le acusa de falta de moral (es un imperativo más fuerte respetar el código de ética, aún si éste no es explícito); el tercero es acusado porque ha sido incapaz de detectar las malversaciones de sus dos colegas (se postula entonces que es más importante despedir a los 
incapaces que a los deshonestos que, por lo menos, sean listos). Cuando se pregunta a los directivos por qué no despiden a los tres, parten en su respuesta de la hipótesis que la empresa no puede quedarse decapitada. Lo interesante es que, como los argumentos propuestos por unos y otros son generalmente insuficientes para justificar su decisión, estos postulados, hipótesis y supuestos, aparecen poco a poco en la discusión para apoyar la posición adoptada; inconscientes al principio, estos imperativos se vuelven conscientes a través del conflicto de opiniones. No son establecidos a priori, como punto de partida para orientar la decisión; surgen como juicios de valor a medida que se desarrolla la discusión, para defender las opiniones en juego.

Los integrantes de estos grupos consideran la situación planteada como demasiado compleja para poder aportar de inmediato una solución, admiten ser incapaces de ponerse de acuerdo en el grupo y estiman que no tienen suficiente información para tomar una decisión. Se produce una parálisis de decisión frente a una situación difícil y, por lo tanto, se prefiere posponer el momento de decidir.

El último esquema mental observado a veces es la "parálisis de decisión". Se puede resumir así: ante lo que llaman la "complejidad del problema", los directivos prefieren no tomar una decisión y proponen crear un comité para hacerlo en el futuro. Consideran que no tienen suficiente información y que les falta tiempo para analizar en profundidad lo que pasa y poder tomar una decisión con todo conocimiento de causa.

Pero si pedir más información sobre una situación es indispensable para tener un conocimiento más amplio de ella, es también peligroso ya que conduce a no tener nun-

\section{Figura 2 - Visión parcial de la situación en función de los lentes de cada actor}

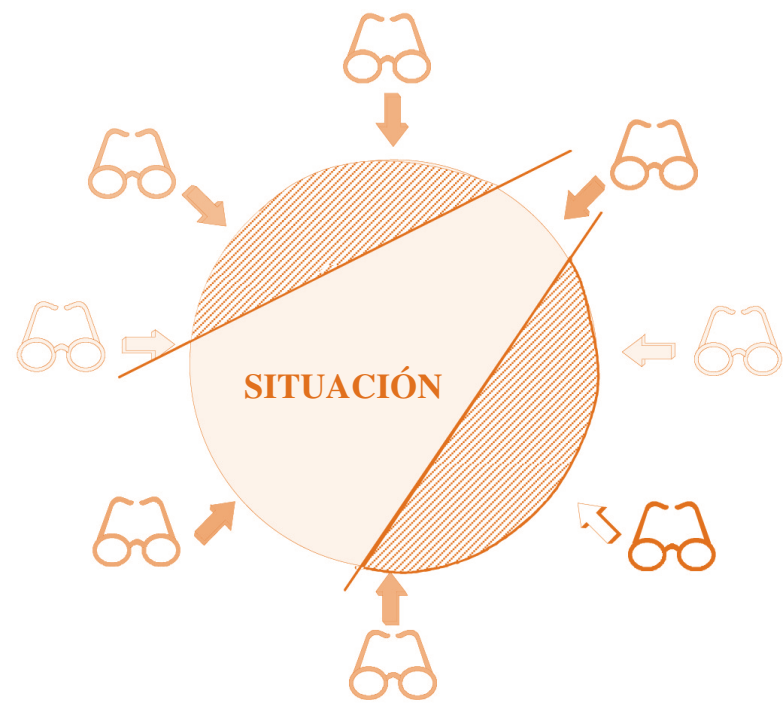

ca suficiente información para poder decidir. Una tendencia muy común es exigir siempre más y mejor información, con el fin de sentirse menos vulnerable en la toma de decisiones.

La misma actitud se observa en relación al tiempo. Los directivos tienden a considerar que hay primero un tiempo de análisis, después un tiempo de decisión y, al final, es el momento de la acción. Piensan, además, que el segundo paso puede realizarse solamente si se conocen todas las posibilidades del primero. Como la tentación de requerir más tiempo de análisis es fuerte, el tiempo de la decisión puede no llegar nunca. Este fenómeno se acentúa cuando se tiene el sentimiento de que existe una decisión mejor que las demás (un estado ideal hacia el cual hay que tender) y que es imperativo utilizar todos los medios para descubrirla.

A menudo, la parálisis de no-decisión se debe al deseo del grupo de directivos de darse el tiempo necesario para encontrar una solución que concilie todos los puntos de vista presentes, pero, generalmente se trata de una huida ante las consecuencias de las posibles decisiones.

La parálisis de no-decisión engendra frustración entre los directivos. Ciertamente, cuando una decisión se toma rápidamente, piensan que el tiempo de análisis fue insuficiente y que la decisión hubiera sido mejor con más información o con más tiempo para tomarla; al no tener suficiente tiempo o información, les parece que el análisis carece de objetividad. Sin embargo, cuando se atrasa la toma de decisión, manifiestan malestar.

\section{POSICIÓN REALISTA VERSUS CONSTRUCTIVISTA}

Frente a una situación compleja, se pueden adoptar dos actitudes teóricas opuestas (ver Figura 3).

La primera actitud posible consiste en considerar que la realidad (el entorno) existe, rodea y se impone al decisor. Por lo tanto, debe intentarse por todos los medios percibirla cada vez más y mejor, con el fin de adaptarse eficazmente a ella. El management tradicional, con su enfoque estratégico, empuja a los directivos a actuar de esta forma: un responsable debe conocer mejor a sus clientes, a sus competidores, a su mercado, a sus costos, etc., para reaccionar a tiempo y decidir con conocimiento de causa una estrategia pertinente y un sistema de información adecuado. Esta actitud se puede calificar de "realista". Según esta manera de ver el mundo (que el management tradicional como disciplina y la educación racional generalmente recibida por los directivos han hecho que se considere como la única aceptable), la meta de un directivo será una mejor identificación y un mejor conocimiento de la realidad, para poder adaptarse mejor a ella.

La segunda actitud que puede tomarse es considerar que la realidad no existe en si, sino que es un producto de la mente del directivo. Por su educación familiar, su 
formación en las aulas, su experiencia, los éxitos obtenidos y los "golpes" recibidos, ha aprendido a construir con su mente el mundo en que vive. No tiende a verlo como es, sino como quiere que sea. Así, un directivo con muchas convicciones llega a creer tanto en ellas que éstas se transforman en su mente en realidad. De la misma forma, un directivo que tenga la convicción de que una estrategia es imposible no la seleccionará, no por que ésta sea en si imposible, sino por que él cree que lo es (Solé, 1996). Esta actitud se puede calificar de "constructivista" (Berger, 1992, Watzlawick, 1978). Según esta manera de ver el mundo, el objetivo del directivo será comprender mejor cómo funciona su mente, es decir, tomar consciencia de sus certidumbres, sus intuiciones, sus postulados, sus imperativos, sus hipótesis y sus supuestos al elaborar un razonamiento, seleccionar y analizar las informaciones que considera pertinentes, tomar una decisión, etc.

Los resultados del dispositivo de investigación que hemos diseñado apoyan más la tesis "constructivista" que la "realista". Un directivo (como cualquier persona, pero quizás más si es un emprendedor, ya que los emprendedores tienen tendencia a salir del mundo convencional para crear algo nuevo) construye mentalmente el mundo que lo rodea y tiene interés en comprender como funciona su mente y cómo reacciona frente a una situación compleja. Sin embargo, no es fácil para un individuo tomar consciencia él solo de sus propios reflejos mentales, de su propio modo de construir una situación. Para entrenarse a eso, le conviene aceptar que:
- hay otros actores (en el sentido que dan a este concepto Crozier Friedberg, 1977) involucrados en la situación;

- cada uno de ellos tiene su propia construcción de la situación;

- crear las condiciones para que los actores puedan confrontar sus puntos de vista facilita la apertura a otras sensibilidades (construcciones) y la toma de conciencia de su propio esquema mental;

- esta actitud permite ampliar la comprensión de la situación.

Por lo tanto, comprender una situación compleja significa identificar en primer término a todos los actores involucrados en ella, evaluar después lo que está en juego para cada uno de ellos, es decir, lo que cada uno gana o pierde al mantenerse la situación actual o efectuando cualquier cambio, decidir finalmente si existe la necesidad de un cambio y cuál debería ser la naturaleza de éste, en función de sus consecuencias en términos de actores y de lo que está en juego para cada uno de ellos.

Para un directivo, comprender una situación consiste primero en identificar a los actores involucrados en ella. Se define a un actor como una persona o un grupo, no necesariamente activo, que tiene algo significativamente en juego en una situación dada. En primera instancia, el directivo elabora la lista más completa posible de los actores internos y externos a la empresa ligados a la situación problemática. Pero, como esta lista es generalmente larga, el directivo tiende a seleccionar

Figura 3 - ¿Percepción o construcción de una realidad?

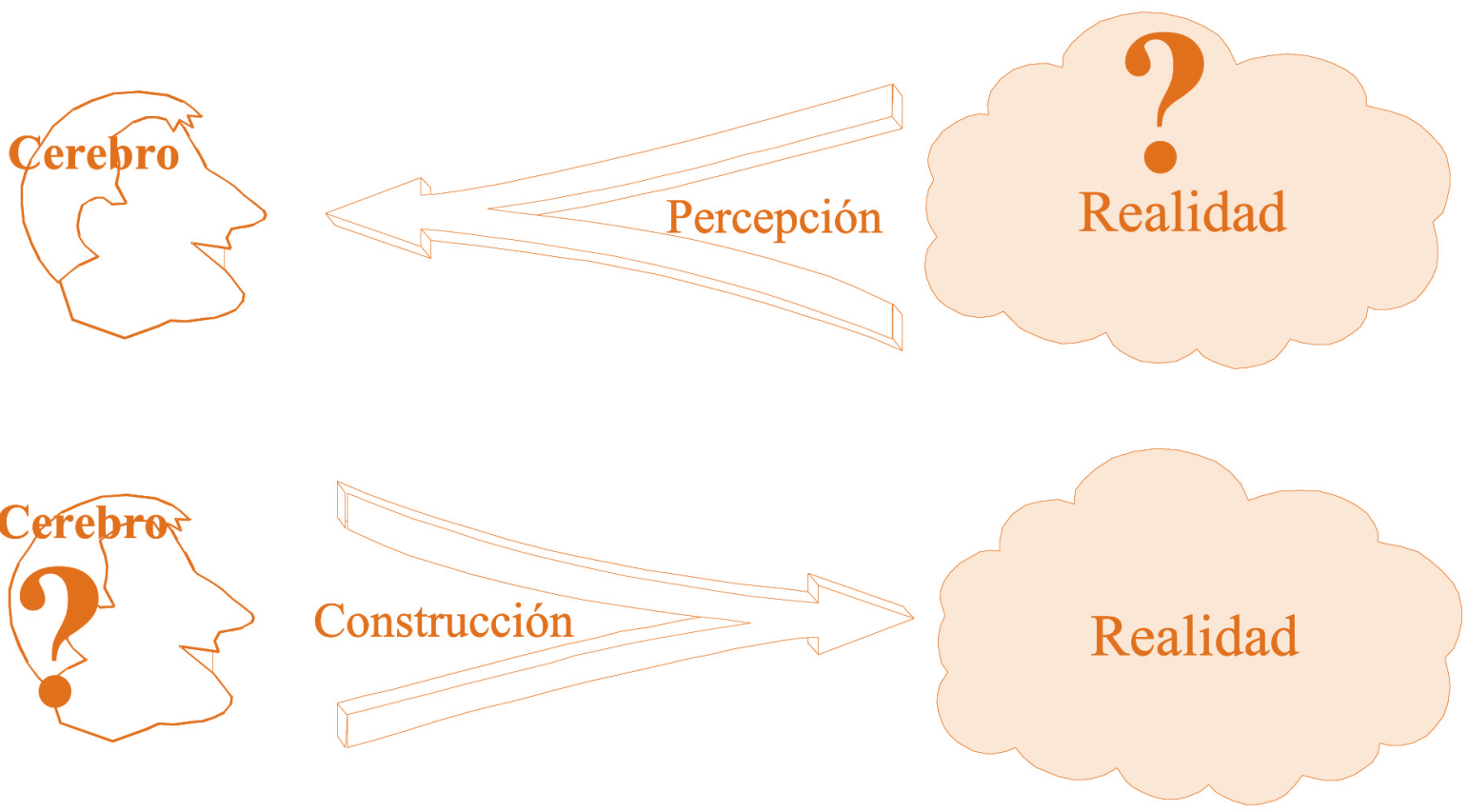


solamente a los actores que considera directa y fuertemente ligados a la situación estudiada. Esta etapa no es neutra; tiene dos limitaciones. Ya que los actores son seleccionados por el dirigente que hace frente a la situación, el directivo puede favorecer a los actores que entran naturalmente en su esquema mental y subestimar a los otros, así, si su visión es esencialmente interna dentro de la empresa, su esquema mental puede cegarlo a ciertos actores externos importantes como los clientes o los proveedores. Además, como la presión del tiempo empuja al dirigente a limitar el número de actores tomados en cuenta, la selección tiene una fuerte probabilidad de ser sesgada. Sin embargo, el dirigente puede siempre preguntarse al terminar la aplicación del enfoque si no hay un actor que no haya sido tomado en cuenta que pudiera poner en duda o invalidar la decisión final tomada.

Comprender una situación para un dirigente es también aceptar que cada actor involucrado la construye en función de lo que tiene en juego en ella. Lo que está en juego para un actor es lo que él puede ganar o perder en la situación problemática estudiada o en cualquier cambio que la afecte. Para algunos autores (Crozier, 1963; Crozier y Friedberg, 1977), lo que está esencialmente en juego para un actor es su poder político respecto a la situación, es decir las zonas de legitimidad que sólo él controla, por ser experto en ellas, y que se vuelven zonas de incertidumbre para los otros actores. Sin embargo, lo que está en juego para un actor puede ser no solamente político (en términos de poder con respecto a una situación), sino también económico, afectivo, ético, religioso, psicológico, etc.

La detección de lo que está en juego para cada uno de los actores seleccionados como significativamente involucrados en la situación presenta una seria limitación. En efecto, el directivo que se propone detectar lo que está en juego para otro actor tiene dos posibilidades: o bien le pregunta directamente qué tiene en juego en la situación o bien se lo imagina él mismo. En la primera opción, hay una fuerte probabilidad de que el actor entrevistado no se descubra, para protegerse. También existe el peligro de que, si llegara a enterarse de lo que esta en juego para un actor, el directivo tenga la tentación de manipularle. En la segunda, que es la opción más común, el dirigente tiene el riesgo de proyectarse sobre el actor y construir lo que, según él, tiene el actor en juego; además, tiende a percibir al actor en términos de alianza, de antagonismo, de poder, de afecto, de principios morales, de reglas organizacionales, etc. Si el directivo prevé realizar un cambio a la situación actual, debe volver a examinar de qué manera la modificación afecta a cada actor involucrado en el juego, es decir, lo que gana y lo que pierde cada uno de ellos. Es muy probable que el directivo no encuentre una solución ideal que a la vez le satisfaga totalmente, así como a todos los otros actores ligados a la situación.
Una solución ideal sería una situación en la que todos ganen. Sin embargo, aún si pudiera encontrarse, esta solución sería momentáneamente ideal y no para siempre. Para resumir, identificar a los actores significa definir el número de lentes que construyen la situación, siendo cada lente lo que está en juego para cada actor. La metáfora de los lentes induce tres reflejos mentales: a) impulsa al dirigente a identificar a los actores que se construyen a su manera la situación problemática; b) le ayuda a definir cuáles son los lentes de cada actor involucrado en la situación para asimilarla, pues esos lentes están en función de lo que tienen en juego en dicha situación; c) lo invita a quitarse sus propios lentes para ponerse los de los demás actores, con el fin de no quedarse preso de los suyos y ampliar su comprensión de la situación. Sin embargo, si la metáfora de los lentes empuja a un directivo a no encerrarse en su propia visión de una situación, tiene como límite la dificultad que tiene cualquier persona y más aún el directivo, por la posición central que ocupa de luchar en contra de sus propias convicciones y certezas.

Podemos preguntarnos si la ayuda de un consultor externo podría facilitar esta toma de conciencia del directivo. Pero no se debe olvidar que el consultor también tiene algo en juego en la situación analizada; como el directivo, tiene un punto ciego.

\section{EL SEGUNDO EXPERIMENTO}

El primer experimento había permitido descubrir que directivos de diferentes países de Europa del Sur y de América Latina desarrollaban reflejos mentales idénticos cuando tenían que tomar decisiones en una situación empresarial compleja. El segundo experimento se diseñó para estudiar si directivos norteamericanos se comportaban de igual forma en la misma situación. Una gran compañía multinacional nos ofreció la oportunidad de realizar este estudio. Se formaron seis grupos de cuatro directivos franceses, españoles, italianos y/o latinoamericanos y dos de cuatro directivos norteamericanos, todos del mismo nivel jerárquico y ocupando puestos de muy similar responsabilidad. Las modalidades prácticas del segundo experimento fueron idénticas a las del primero: se utilizó el mismo caso y se organizaron las mismas condiciones de discusión.

Nuestra hipótesis era que todos los grupos iban a desarrollar los mismos reflejos mentales. No se verificó. Los resultados obtenidos fueron muy diferentes entre los grupos compuestos de norteamericanos y los otros.

- En los dos grupos norteamericanos, el proceso de discusión duró poco tiempo y la decisión fue tomada en menos de 15 minutos. Los miembros de estos grupos no manifestaron ningún desconcierto metodológico, pero fueron directamente a la solución. 
Su decisión fue la misma: despedir al responsable de producción por delito e informar inmediatamente al director general. Cuando se preguntó a los integrantes de los dos grupos por qué habían tomado esta decisión tan rápidamente, defendieron todos dos argumentos: a) el responsable de producción ha infringido un principio ético básico: ha cometido un delito y debe ser despedido al instante; b) cuando algo anda mal, uno debe prevenir inmediatamente, sin vacilar un segundo, a su superior jerárquico, cualquiera que sea la reacción que se espere de él. Uno debe asumir enteramente sus errores.

- Ninguno de los dos grupos manifestó que el objetivo del director general fuese inalcanzable, pero tampoco ninguno de ellos lo consideró alcanzable.

- Los seis grupos compuestos de latinos utilizaron las dos horas acordadas para tomar su decisión, dividiéndose tanto en el diagnóstico de la situación como en la selección de la solución propuesta y discutiendo mucho entre si sus diferentes opiniones. Manifestaron vivir un fuerte desconcierto metodológico. Los seis grupos consideraron el objetivo del director general inalcanzable y propusieron revisar fuertemente a la baja dicho objetivo. Además, si bien todos sugirieron una solución, no llegaron a la misma; las soluciones propuestas fueron muy variadas. Todos decidieron elaborar un plan de reajuste antes de entrevistarse con el director general; dos grupos propusieron sin embargo, presentarle su renuncia. Un grupo despidió al gerente de ventas, otro al responsable de producción y un tercero al contralor de gestión.

\section{CONCLUSIÓN}

¿Cuáles son las enseñanzas que podemos sacar de estos dos experimentos? Primero, debemos ser sumamente prudentes en cuanto a los resultados obtenidos en el segundo experimento, ya que han sido solamente dos los grupos de directivos norteamericanos observados. Por el contrario, pensamos que, dado el tamaño de la muestra de directivos latinos, las conclusiones sacadas del primer experimento son muy representativas: ante situaciones complejas, los directivos latinos - tanto europeos como americanos - suelen manifestar un cierto desconcierto metodológico, tienen la mentalidadsolución, se enfrentan mediante la lógica del conflicto de opiniones y pueden tender a la parálisis de decisión. Segundo, pensamos que ahora podemos proponer una hipótesis opuesta a la que habíamos sugerido anteriormente. La nueva puede formularse así: frente a situaciones complejas en las que hay que tomar una decisión bajo presión, los directivos latinos y sus homólogos no desarrollan exactamente los mismos reflejos mentales que los norteamericanos: son menos sensibles a metodologías lineales y directas; son menos estrictos en la aplicación de principios éticos; se muestran más complejos, a veces maquiavélicos, en su toma de decisiones y aprecian mucho más el debate, aún si se trata más de discutir opiniones que de confrontar con calma puntos de vista. Cuarto y último punto: todos tienen la mentalidad-solución. ¿Será una deformación engendrada por la administración de empresa moderna, sus recetas y sus modas? $\bigcirc$ algunos de los numerosos actores de la situación; b) el mismo autor del caso ha seleccionado parcialmente las informaciones o hechos incluidos.

3. Algunos de estos trabajos fueron realizados en el marco de uno de los programas de educación permanente de la Escuela de Administración de Empresas de la Fundação Getulio Vargas.

4. Estos reflejos cognoscitivos han sido desarrollados en FIOL, Michel, Administración de Empresas: necesidad de rediseñar la educación permanente, El Mercado de Valores, jun. 1999, p. 34-44.

\section{REFERENCIAS}

BERGER, Peter, LUCKMANN, Thomas. La construction sociale de la réalité. Paris : Méridien Klincksieck, 1992. Chap. 1, p. 31-68.

CROZIER, Michel. Le phénomène bureaucratique. Paris : Les Editions du Seuil, 1963.

CROZIER, Michel, FRIEDBERG, Erhard. L'acteur et le système. Paris : Les Editions du Seuil, 1977.
FIOL, Michel et al. Le manager: histoire d'une idée. Informe de Investigación, HEC Executive Management Center, Mars, 1999. 412 p.

SOLÉ, Andreú. La décision: production de possibles et d'impossibles. In: CAZAMIAN P., HUBAULT, F. NOULIN, M. Traité d'ergonomie. Toulouse : Octares editions, 1996.
WATZLAWICK, Paul. L'invention de la réalité: contributions au constructivisme. Paris : Le Seuil, 1978.

WILLIAMSON, Oliver. Corporate control and business behavior. Englewood Cliffs, NJ : Prentice Hall, 1970. 